\title{
Criminologie
}

\section{Haute et basse police après le 11 septembre (2008)}

\section{Jean-Paul Brodeur et Frédéric Ocqueteau}

Volume 44, numéro 1, printemps 2011

Jean-Paul Brodeur, d’hier à aujourd'hui

URI : https://id.erudit.org/iderudit/1001608ar

DOI : https://doi.org/10.7202/1001608ar

Aller au sommaire du numéro

Éditeur(s)

Les Presses de l’Université de Montréal

ISSN

0316-0041 (imprimé)

1492-1367 (numérique)

Découvrir la revue

Citer cet article

Brodeur, J.-P. \& Ocqueteau, F. (2011). Haute et basse police après le 11 septembre (2008). Criminologie, 44(1), 225-245.

https://doi.org/10.7202/1001608ar

\section{Résumé de l'article}

La distinction entre la haute police (police politique) et la basse police (police criminelle ordinaire) a accru sa pertinence dans le prolongement des attentats terroristes du 11 septembre 2001 (9/11). Ce texte reprend le contenu du paradigme de la haute police proposé en 1983 et répond à certaines critiques qui lui ont été récemment adressées. Nous proposons d'abord une remise à date des caractéristiques de la haute police. Celle-ci est considérée comme une police d'absorption, d'amalgame des pouvoirs au profit du pouvoir exécutif, de protection de l'État et d'infiltration de la société par des indicateurs de police. Nous montrons ensuite que les différentes commissions d'enquête qui se sont penchées sur les lacunes de l'action policière et des services de renseignement dans la prévention des attentats du 9/11 ont trouvé que la distinction entre la haute et la basse police était encore plus profonde qu'on ne l'avait anticipé. Dans une troisième partie du texte, la question de l'existence d'une haute police privée est abordée. Bien que l'existence d'une haute police privée ne puisse maintenant être remise en question, cette haute police privée ne possède qu'en partie les traits qui définissent le modèle initial de la haute police publique. Dans la dernière partie, le contraste entre haute et basse police est examiné à la lumière de sa résonance symbolique.
Ce document est protégé par la loi sur le droit d'auteur. L'utilisation des services d’Érudit (y compris la reproduction) est assujettie à sa politique d'utilisation que vous pouvez consulter en ligne.

https://apropos.erudit.org/fr/usagers/politique-dutilisation/ 


\section{Haute et basse police après le 11 septembre}

Jean-Paul Brodeur $\left(2008^{\star}\right)$

\section{Note introductive}

Cet article témoigne de deux caractéristiques majeures de la démarche intellectuelle de Jean-Paul Brodeur: d'une part, sa capacité à se tenir au plus près des avancées des sciences dans le vaste domaine du policing; d'autre part, une heureuse aptitude à remodéliser ses propres schémas d'analyse à mesure que l'accumulation de données disponibles l'y oblige.

Il était donc assez fatal que l'évolution du monde de «l'après 11 septembre» aille non seulement révolutionner les approches criminologiques de la sécurité internationale en général, mais que, tôt ou tard, sa célèbre distinction heuristique entre haute et basse polices fût ébranlée, exigeant de sa part un toilettage en profondeur.

L'occasion de jeter les bases d'une nouvelle réflexion à ce sujet me semble avoir été favorisée par la convergence de plusieurs préoccupations souterraines de Jean-Paul Brodeur. On sait que son bagage philosophique l'avait conduit à porter une attention extrême au travail d'H. L'Heuillet sur la «haute police et la basse politique» (2001). Et plus récemment, que son insatiable curiosité l'avait amené à rencontrer dans la thèse novatrice des Irlandais C. O'Reilly et G. Ellison (2006), une avancée majeure. Ces chercheurs ont en effet été les tout premiers à revisiter de fond en comble la notion de «haute police» à la lumière des tendances à la privatisation des services de renseignements occidentaux. Comme par ailleurs, Jean-Paul Brodeur paraissait de plus en

${ }^{\star}$ Criminologie, 41 (1), 133-151.

Criminologie, vol. $44, \mathrm{n}^{\circ} 1$ (2011) 
plus insatisfait de la thèse influente sur les policiers comme «travailleurs du savoir», il avait désormais toutes les cartes en mains pour jeter les bases de cet article, profond, dense et lumineux, pour célébrer, comme il se devait, le $40^{\mathrm{e}}$ anniversaire de la revue Criminologie ${ }^{1}$.

L'article ultra concentré reste néanmoins extrêmement pédagogique: dans sa première partie, sont évoqués les quatre éléments constitutifs d'une définition provisoire de la nouvelle «haute police». Mais il est également dialectique: tandis que la deuxième partie inventorie en effet des éléments empiriques rendant problématique la tendance au mouvement d'intégration de la haute et de la basse police, la troisième propose d'en dépasser les freins en recourant à la métaphore de «l'hybridation de la haute police». Dans ce passage, Jean-Paul Brodeur ajoute à la thèse des auteurs précités, un enjeu à ses yeux sous-évalué: celui de la protection de «clients» concurrents faisant l'objet de menées subversives. Cet ajout me paraît capital et demandera sans aucun doute à être creusé par les analystes futurs. Il leur faudra en outre tenir compte de la nature du statut (public ou privé) des clients eux-mêmes en compétition pour différencier l'évaluation de l'impact et des performances empiriques de ladite «haute police hybride» dédiée à la défense de leurs intérêts. Dans la quatrième partie, Jean-Paul Brodeur redevient le citoyen que les audaces de sa science ne l'ont jamais empêché de rester. Il concède à l'État - agirait-il dans l'illégalité institutionnalisée, comme le feraient de manière plus débridée les entreprises de la haute police privée -, sa faculté de conserver le monopole du pouvoir de protéger l'ensemble des citoyens de son territoire. Autrement dit, de bénéficier d'une légitimité bien supérieure à ses yeux que celle de ces entreprises, seraient-elles devenues aussi - voire plus - puissantes que les États eux-mêmes.

Frédéric Ocqueteau ${ }^{\star \star}$

RÉSUMÉ - La distinction entre la haute police (police politique) et la basse police (police criminelle ordinaire) a accru sa pertinence dans le prolongement des attentats

1. Signalons que dans son traité posthume, J-P. Brodeur est revenu sur la question de la haute et de la basse police (chapitre 7), et sur la privatisation de la «haute police» (chapitre 8, spécialement, p. 288-293). Cf. Brodeur, J.-P. (2010). The policing Web. Oxford: Oxford University Press.

${ }^{\star \star}$ Centre national de la recherche scientifique (CNRS) ocqueteau@cesdip.fr 
terroristes du 11 septembre 2001 (9/11). Ce texte reprend le contenu du paradigme de la haute police proposé en 1983 et répond à certaines critiques qui lui ont été récemment adressées. Nous proposons d'abord une remise à date des caractéristiques de la haute police. Celle-ci est considérée comme une police d'absorption, d'amalgame des pouvoirs au profit du pouvoir exécutif, de protection de l'État et d'infiltration de la société par des indicateurs de police. Nous montrons ensuite que les différentes commissions d'enquête qui se sont penchées sur les lacunes de l'action policière et des services de renseignement dans la prévention des attentats du 9/11 ont trouvé que la distinction entre la haute et la basse police était encore plus profonde qu'on ne l'avait anticipé. Dans une troisième partie du texte, la question de l'existence d'une haute police privée est abordée. Bien que l'existence d'une haute police privée ne puisse maintenant être remise en question, cette haute police privée ne possède qu'en partie les traits qui définissent le modèle initial de la haute police publique. Dans la dernière partie, le contraste entre haute et basse police est examiné à la lumière de sa résonance symbolique.

MOTS-CLÉs - Haute police, police politique, basse police, police criminelle, commissions d'enquête.

\section{Introduction ${ }^{1}$}

La distinction que j'ai faite entre haute et basse police (Brodeur, 1983), suivant le modèle français traditionnel, a suscité intérêt (Marenin, 1996: 8; L'Heuillet, 2001 : 39; Manning, 2003: 41 et 201 ; Manning, 2005 : 24) et critiques (Anderson et al., 1995: 169; O'Reilly et Ellison, 2006: 643-45). Après avoir réintroduit cette distinction classique, je l'ai appliquée à l'analyse de diverses formes de police politique (Brodeur, 2003 ; Brodeur et Dupeyron, 2003), tout en mettant à jour la notion de haute police, et ce, en tenant compte de l'évolution récente dans le domaine. Enfin, j'ai avancé que les agences de haute police étaient de plus en plus impliquées dans des activités de basse police et vice-versa (Brodeur, 2000/2005a). Par ailleurs, Brodeur et Leman-Langlois (2006) ont aussi montré comment, sous l'administration de George W. Bush, le gouvernement des États-Unis confie davantage la protection de la sécurité nationale à des entreprises privées.

Cet article se divise en quatre parties. D’abord, je synthétise la littérature existante sur la nature de la haute police, en mettant l'accent sur la période post-1983. Ensuite, je remets en question l'intégration de la haute et de la basse police. Puis, j'expose toute l'étendue du phénomène de la privatisation de la haute police. Enfin, je contraste haute et basse police par rapport à ce qu'elles symbolisent.

1. Traduit de l'anglais par Marie-Ève Racette et révisé par Geneviève Ouellet. 


\section{Haute et basse police : définition et méthode}

La distinction faite entre haute et basse police est asymétrique. Lorsque je l'ai proposée (Brodeur, 1983), je tentais de mettre en exergue un type de services policiers très différents des services qui sont généralement assurés par des agents en uniforme; services qui étaient, et sont toujours, négligés par les chercheurs, malgré leur importance croissante. Les agences qui assurent ces services appartiennent à la «communauté du renseignement» et j'ai qualifié leurs activités de «haute police», empruntant l'expression française. Cette distinction mettait donc l'accent sur la haute police, sans offrir une description précise de la «basse police». Cet article vise à combler cette lacune.

Samuel Johnson, dans son Dictionary of the English Language, reconnaît que le mot «police» est un emprunt du français et qu'il signifie «the regulation and government of a city or country, so far as regards the inbabi$\operatorname{tants}^{2} »$ (Johnson, 1806: ii, «police»). Cette définition correspond bien à celle proposée à l'impératrice autrichienne Marie-Thérèse par J. C. P. Lenoir, l'un des plus connus des lieutenants généraux de police de France: la police est «la science de gouverner les hommes et de leur faire du bien» (paraphrase) (Lenoir, 1779: 34). À l'origine, le mot «police» était synonyme du mot «gouvernance ${ }^{3} »$. Le ministre de la Police de Napoléon, Joseph Fouché, qui a articulé le concept de haute police, avait fait une distinction explicite entre l'établissement d'un ordre politique («faire la police») et l'accomplissement de tâches et actions policières («faire de la police») (Madelin, 1930: i. 490). Alors que la distinction contemporaine se fait entre la police et les activités policières (Reiner, 1994: 715), il y avait à l'époque une construction en trois volets : (1) «LA police» - le régime politique dominant et l'ordre établi; (2) «les activités policières» (correspondant à l'expression anglaise policing), soit les actions entreprises pour renforcer directement le régime politique et celles qui visent indirectement ce but par la prévention et la répression de crimes et de désordres - bref, la production de la sécurité; (3) «les policiers», soit les membres des organisations accomplissant

2. «La régulation et le gouvernement d'une ville ou d'un pays, tels qu'appliqués à leurs habitants» (traduction).

3. Le mot français "police» vient du grec «polis», qui veut dire «ville» et qui a gardé son sens original en français. Pour les Français, comme pour Johnson, «la police» était un concept abstrait qui n'avait aucun rapport avec une organisation ou avec des personnes. 
les activités policières, incluant les agents officiels de l'État et une foule d'agents privés (en gros, les informateurs). Les agents de gouvernance - ceux qui défendaient «LA police» (l'ordre politique) du royaume effectuaient des tâches de "haute police», puisqu'ils étaient le bras exécutif du roi. Les autres, qui s'affairaient à une myriade de besognes liées à la sécurité commune - ce que Fouché appelait «la police des lampadaires» (sous lesquels se postaient les prostituées) - effectuaient de la «basse police» et étaient responsables devant les tribunaux. Donc, haute et basse police, à l'origine, désignaient une échelle de prestige en fonction de la proximité des autorités policières aux assises du pouvoir. Aujourd'hui, des agences comme le MI5 et le MI6 au Royaume-Uni, les unités de surveillance politique du FBI et la CIA ${ }^{4}$ aux États-Unis, la DST (Direction de la surveillance du territoire) de France ou encore le Service canadien de renseignement de sécurité (SCRS) sont des exemples de haute police.

La haute police se définit par quatre éléments de base (Brodeur, 1983 : 513-514; Brodeur, 2006: 181-190; voir aussi O’Reilly et Ellison, 2005: 643-645):

A. C'est une police d'absorption. Les agences de haute police recueillent des données, les transforment en renseignement (information analysée) et évaluent les menaces, divulguent les renseignements au besoin, les sauvegardent dans divers formats pendant une période déterminée et les suppriment lorsqu'ils perdent de leur utilité. Il existe deux différences fondamentales entre le renseignement de haute police (renseignement de sécurité) et de basse police (renseignement criminel). D'abord, ils n'ont pas la même portée. Les forces policières se limitent à recueillir les renseignements nécessaires à l'établissement d'une preuve. Les services de sécurité, par contre, ont un appétit insatiable pour l'information. Par exemple, le World Factbook de la CIA, accessible sur Internet, donne des renseignements de base sur presque tous les pays du monde, en mettant l'accent sur leurs problèmes de criminalité et de sécurités. La seconde différence concerne un type de renseignement appelé, en anglais, «actionable intelligence». Il s'agit de renseignements qui pousseront une agence à passer de la surveillance passive à l'intervention, par exemple à l'arres-

4. Il existe officiellement 16 agences de ce genre aux États-Unis. $80 \%$ du budget du renseignement revient au Pentagone. On trouvera la liste des agences de haute police étatsuniennes dans United States (2004: 406-07, rapport de la commission 9/11).

5. Voir https://www.cia.gov/cia/publications/factbook/index.htlm 
tation et à l'inculpation d'un suspect ${ }^{6}$. Bref, pour la police, le renseignement n'est qu'un moyen pour établir une preuve, alors que les agences de sécurité ont, au contraire, une tendance beaucoup plus prononcée à absorber le renseignement et à intervenir uniquement lorsqu'il n'y a pas de solution de rechange.

B. L'amalgamation des pouvoirs. On fait traditionnellement la distinction entre les pouvoirs législatif, judiciaire et exécutif qui, dans les démocraties, sont indépendants les uns des autres. Dans le modèle Westminster, où les ministres du Cabinet siègent aussi au Parlement, il y a une moins grande distinction entre les organes exécutif et législatif qu'aux ÉtatsUnis, où l'on trouve le système de "freins et contrepoids» (checks and balances). Cependant, sans égard au modèle choisi, toutes les démocraties réprouvent l'ingérence du gouvernement dans les tribunaux. Il n'en va pas de même pour la haute police. Sous les monarchies continentales d'Europe, le magistrat de police jouissait des trois pouvoirs: il pouvait établir des lois pénales comportant jusqu'à la peine de mort; il pouvait aussi présider à des procès; enfin, il pouvait exercer, par définition, toutes les formes de pouvoir exécutif. En fait, du XviI ${ }^{e}$ au xIX $^{\mathrm{e}}$ siècles, le concept du «coup» politique avait une signification qui contredit son sens actuel: il s'agissait d'une action décisive de l'État contre ses ennemis et non d'une action perpétrée contre l'État par ses opposants, dans le but de modifier le régime en place (L'Heuillet, 2001 : 47, citation de Gabriel Naudé, 1639/1988). Le magistrat de haute police était responsable de la gouvernance par coups exécutifs. Dans une des sections de cet article, j'avancerai que nous sommes aujourd'hui témoins de la résurgence de ce mode de gouvernance depuis le 11 septembre.

C. La protection de la sécurité nationale. C'est la raison d'être de la haute police. Le mandat de nombreuses agences de renseignement de sécurité stipule que leur principal objectif est d'assurer la sécurité de l'État. Il existe deux variantes très différentes de cet aspect. Dans les démocraties, les agences de haute police défendent les institutions politiques et le cadre constitutionnel du pays. Pour ce qui est des gouvernements non démocratiques, la haute police est dévolue à préserver un régime politique particulier, qui peut consister en l'hégémonie d'un parti politique,

6. Ajoutons que dans certaines agences de haute police - par exemple, la CIA, le Shin Bet et le Mossad d'Israel, ou la DGSE de France - les renseignements «actionnables» déclenchent des opérations spéciales de neutralisation de l'ennemi. 
voire d'un dictateur. Il importe de faire la distinction entre ces variantes de sécurité nationale, afin d'éviter de tomber dans le sophisme de gauche selon lequel les services de renseignement sont, par leur nature même, incompatibles avec la démocratie. Il faut cependant bien comprendre que l'objectif immédiat de la haute police n'en reste pas moins la protection de l'appareil d'État (par exemple, défendre le chef d'État de toute tentative d'assassinat), bien que défendre l'État signifie, par extension, protéger les citoyens (par exemple, contre le terrorisme).

D. L'utilisation d'informateurs. En jargon policier, un informateur est appelé une «source humaine», ce qui, lorsque le paradigme de la haute police a été développé, était le principal instrument de surveillance clandestine. Il existe aujourd'hui tout un arsenal d'outils technologiques à cet effet (Marx, 1988: chapitre 10). Tous nos sens naturels - la vue, l'ouie, l'odorat, le toucher (détecteurs de mensonges) et même le goût (dispositifs de détection des poisons) - ont désormais leur réplique technologique. Toutefois, en dépit de l'existence d'une panoplie d'outils de surveillance technologique, j'avance que l'utilisation de sources humaines et d'infiltrateurs est toujours l'essence même de la haute police. Comme le montrent bien les archives de la Stasi d'Allemagne de l'Est $^{7}$, récemment rendues publiques, l'infiltration tous azimuts par des sources humaines est l'aboutissement de la haute police. Non seulement il s'agit de l'instrument de surveillance le plus intrusif, mais il est également le plus destructeur du tissu social, puisqu'il se nourrit de trahison et engendre la suspicion mutuelle et la démoralisation (Funder, 2003).

J'avais élaboré ces idées à partir de recherches menées dans les dossiers de services de renseignement canadiens, documents auxquels j'avais eu accès alors que j'étais directeur de recherche pour une commission d'enquête québécoise possédant des pouvoirs judiciaires. À ce jour, je continue de travailler auprès de diverses commissions qui enquêtent, directement ou indirectement, sur la haute police. Bien qu'il soit possible de faire des recherches sur la haute police sans avoir cet accès privilégié, il faut, dans ce cas, veiller à toujours maintenir les plus hautes normes d'exactitude et éviter de se fier à des extrapolations tirées des seuls ouvrages publiés. On pourra inclure divers types d'activités policières sous la catégorie «haute police» selon qu'elles présentent

7. De l'Allemand Staatsicherheit, «sécurité de l'État». 
plusieurs des caractéristiques énoncées ci-dessus. Simplement assimiler tout «travail du savoir» accompli par la police à de la haute police fait en sorte que la notion perd de sa valeur heuristique.

\section{L'intégration problématique des haute et basse polices}

Il ne peut être contesté qu'il y a eu, dans l'intervalle entre la tombée du mur de Berlin en 1989 et les attaques terroristes du 11 septembre 2001, d'importantes initiatives - particulièrement de la part des agences de renseignement en quête d'un nouveau mandat - pour fusionner haute et basse police. Depuis la fin de la Guerre froide, les agences de renseignement de sécurité ont investi des domaines qui étaient traditionnellement du ressort des agences d'application de la loi, tel le crime organisé (Brodeur, 2000/2005a ; voir aussi Anderson et al., 1995 : 173). Pour leur part, les forces de police ont tenté de se doter d'unités de renseignement criminel centralisées et ont accru leur implication dans la lutte au crime transnational (Anderson et al., 1995: 168-70). La situation a changé de façon draconienne au tournant du xxi ${ }^{e}$ siècle, avec l'apparition du terrorisme global de masse, qui a affligé non seulement les États-Unis (9/11), mais aussi l'Indonésie (Bali, en octobre 2002, avec plusieurs victimes australiennes), l'Espagne (Madrid, en mars 2003), le Maroc (Casablanca, en mai 2003), l'Arabie saoudite (al-Khobar, en mai 2004) et le Royaume-Uni (Londres, en juillet 2005). Bien qu'il soit encore trop tôt pour évaluer le véritable impact des attaques à la bombe de Londres de juillet 2005, on sait d'ores et déjà qu'elles ont donné lieu au Terrorism Act de 2006, qui prolonge la période de détention préventive, sans qu'aucune accusation ne soit portée, de 14 à 28 jours ${ }^{8}$.

Aux États-Unis, les nombreuses commissions d'enquête chargées de comprendre pourquoi les diverses agences de police étatsuniennes avaient échoué dans la prévention des attaques du 11 septembre, ont produit des conclusions qui ont souligné les différences entre haute et basse police. Au Canada, deux commissions d'enquête gouvernementales en sont venues aux mêmes conclusions:

8. En ce qui concerne la diffusion de l'information, il semble que les forces policières britanniques n'aient guère mieux réussi que leurs homologues étatsuniennes. L'on sait maintenant que Sir Ian Blair, le directeur de la Metropolitan Police, avait été tenu dans l'ignorance pendant au moins 24 heures sur les véritables circonstances de la mort par balles de Jean Charles De Menezes. 
A. La cloison anti-contamination. Dans toutes les enquêtes du gouvernement des États-Unis sur les ratés du renseignement et des forces de l'ordre qui ont mené aux attaques terroristes du 11 septembre, aucune analyse n'est plus incisive que celle proposée dans les «Additional Views» du sénateur Richard C. Shelby, dans un rapport préparé par le US Senate Select Committee on Intelligence (SSCI: United States, 2002). On y trouve le témoignage d'un des agents du FBI impliqué dans l'enquête sur l'attaque à la bombe de 2001 contre le navire étatsunien USS Cole, qui évoqua l'existence d'un «mur» séparant les enquêtes criminelles policières du renseignement de sécurité, afin de préserver «against contaminating criminal investigators with intelligence information ${ }^{9}$ » (cité dans United States, 2002 : 51). Shelby y cite également un ancien directeur de la National Security Agency, qui affirme que les «flics» ne peuvent faire le travail des «espions» (United States, 2002: 74). Le sénateur conclut que: "Intelligence analysts would doubtless make poor policemen, and it has become very clear that policemen make poor intelligence analysts ${ }^{10}$ » (United States, 2002: 62). Son diagnostic des graves lacunes du FBI dans le domaine $\mathrm{du}$ renseignement anti-terroriste fut confirmé par les recommandations de la commission du 11 septembre (United States, 2004: 400). Au Canada, l'existence d'un «mur» entre enquête criminelle et renseignement de sécurité fut incontestablement confirmée par les remarques que l'ancien commissaire de la Gendarmerie royale du Canada (GRC), Giuliano Zaccardelli, fit au sujet de la police du renseignement criminel (intelligence-led policing [ILP], concept principalement développé au Royaume-Uni. Voir National Centre for Police Excellence, 2005). Lors d'un discours public prononcé en 2005, Zaccardelli remarqua que l'ILP "reeks of secret service, spy agency work - the capital " $I$ " in "Intelligence ${ }^{11}$ » (Zaccardelli, 2005).

B. Le renseignement vs la preuve. Le principal différend opposant les agences d'application de la loi et les services de renseignement de sécurité serait ce que le sénateur Shelby a appelé la «tyrannie de la cause judiciaire» (United States, 2002: 62). Toutes les agences d'application de la loi sont organisées de manière à accuser des suspects et à

9. «les enquêteurs policiers contre la contamination par le renseignement» (traduction).

10. «Les analystes du renseignements feraient de piètres policiers et il est désormais évident que les policiers font de très piètres analystes du renseignement.» (Traduction)

11. «empeste le service secret, l'espionnage - le Renseignement avec un R majuscule» (traduction). 
les soumettre à un tribunal. En raison de la nature publique des procès criminels, les agences de renseignement rechignent à divulguer de l'information aux organisations policières, de peur que leurs sources et méthodes soient étalées au grand jour. Au Canada, cette friction entre haute et basse police est persistante. Depuis 1969, il n'y a pas un seul corps gouvernemental ayant examiné les relations entre la communauté canadienne du renseignement et la GRC qui n'ait pas explicitement fait référence au divorce entre renseignement secret et preuve publique. Ce divorce fut tristement illustré lors des deux attentats à la bombe de 1985 contre des vols d'Air India et de CP Air en partance d'aéroports canadiens. Attribués à des terroristes sikhs, ces attentats, qui ont fait 331 victimes, sont de loin les actes terroristes les plus meurtriers de l'histoire du Canada. Dès le début de l'enquête policière, des agents du SCRS ont détruit des enregistrements audio qui auraient pu fournir des preuves cruciales à la police, afin de protéger l'identité de leurs informateurs. Ce conflit persistant opposant SCRS et GRC a été identifié comme étant à la source de l'échec des agences policières canadiennes dans la résolution de cette affaire. Une commission visant cette enquête bâclée a été créée en 2006: la Commission d'enquête relative aux mesures d'investigation prises à la suite de l'attentat à la bombe commis contre le vol 182 d'Air India. Le mandat de la Commission est d'expliquer «[comment le gouvernement canadien peut-il relever le défi [...] de concilier efficacement le renseignement de sécurité et la preuve qui peut être utilisée en matière criminelle». Il m’a d'ailleurs été demandé, par la Commission, de présenter un rapport sur cette question (entre autres).

C. Interruption et encerclement. Les exigences conflictuelles entre production de renseignement et divulgation de la preuve s'insèrent dans l'incompatibilité plus fondamentale entre la culture professionnelle d'une force de police et celle d'un service de renseignement. La culture d'un service de renseignement, qu'il soit policier ou civil, fut décrite par les commissions d'enquête Keable et Duchaîne, pour lesquelles j'étais directeur de recherche, comme une culture de l'encerclement (Québec, 1981a et b; Brodeur, 1981). Entre novembre 1970 et 1973, l'unité anti-terroriste de la police de Montréal avait transformé le Front de libération du Québec (FLQ) en une véritable colonie policière, l'infiltrant de nombreux informateurs. Toutefois, elle se limita à surveiller ses crimes relativement mineurs (par exemple, un attentat à la bombe incendiaire contre un édifice inhabité) tout en canalisant le 
groupe, par l'entremise des informateurs, vers une orientation progressivement moins radicale. Plus de vingt ans plus tard, le Comité de surveillance des activités de renseignement de sécurité (CSARS), responsable du SCRS, a redécouvert cette culture de l'encerclement des services de sécurité: "We are also cognizant of the danger that in destroying one group, as opposed to watching it, another one which is worse may be created ${ }^{12} »($ CSARS, 1994: section 13.11). La police, quant à elle, traite chaque affaire de façon isolée en vue d'interrompre les activités criminelles. Ces interruptions sont parfois durables, voire permanentes, mais dans la plupart des cas elles sont seulement temporaires. La culture de l'encerclement des services de renseignement se prête aux théories du complot et à la mythologie. Pourtant, elle est bien réelle ${ }^{13}$.

\section{L'hybridation de la haute police}

Malgré les études innovatrices de Shearing et Stenning (1981) sur la croissance de l'industrie de la sécurité privée et l'introduction du concept des organisations policières hybrides (Johnston, 1992), la question de la haute police privée mériterait que la recherche s'y attarde davantage. Le rôle historique des agences de sécurité privée, telle Pinkerton, dans la haute police des relations de travail aux États-Unis au XIX ${ }^{e}$ siècle, est déjà pleinement reconnu. Il suffit de penser à l'utilisation de ces agences pour écraser le mouvement militant Mollie Maguire (Weiss, 1986). Dans une nouvelle mouture du paradigme de haute police, Brodeur et Leman-Langlois (2006: 179 14 ) ont noté la tendance à l'impartition d'opérations de surveillance à des entreprises privées aux États-Unis et ailleurs, ainsi qu'une réaffirmation de l'idéo-

12. «Nous sommes également conscients de la possibilité que la destruction, plutôt que la surveillance d'un groupe, pourrait permettre la création d'un second, encore plus dangereux.» (Traduction)

13. Dans son rapport spécial, le juge américain Charles Breitel documente l'étendue de l'infiltration de deux partis trotskistes par le FBI (Breitel, 1980), dont le leadership même comportait des informateurs du FBI. Voir aussi l'incontournable article de Gary Marx (1974). En 2006, la GRC et le SCRS ont arrêté 17 personnes à Toronto - dont quatre adolescents - soupçonnés de planifier une attaque à la bombe. Les reportages médiatiques, jamais démentis par la GRC ni le SCRS, voulaient que celui chargé d'obtenir les explosifs était un informateur de la GRC. Aucun des suspects n'a encore été traduit en justice.

14. Larticle fait mention, en particulier, des entreprises privées suivantes: Raytheon, Syntek Technologies, Booz Allen, Hamilton Inc., Hicks and Associates, Microsoft, Intel et Veridian Corporation (Brodeur et Leman-Langlois, 2006: 179). 
logie de défense du régime politique dominant plutôt que celle du citoyen. La haute police continue donc de se caractériser par son biais pro-étatique. Cependant, O'Reilly et Ellison (2003 : 647) avancent que cette orientation discriminatoire de la haute police ne joue pas exclusivement en faveur de l'État: «while public high policing protects against the subversion of the state, private high policing protects against the subversion of the client $^{15}$ » (je souligne). Cette affirmation est ambiguë, car elle néglige de mentionner qu'il n'y a qu'un État à protéger, alors qu'il y a plusieurs clients qui peuvent avoir des intérêts conflictuels. Tout en admettant le besoin de reconnaitre l'hybridation public-privé de la haute police, j'aimerais présenter trois autres arguments:

A. Données et symboles. La nouvelle conceptualisation de la haute police offerte par O'Reilly et Ellison s'inscrit dans une nouvelle tendance, dans la littérature, qui consiste à souligner la croissance de la sécurité privée. Cette croissance présenterait plusieurs conséquences: multilatéralisation (Bayley et Shearing, 2001), gouvernance nodale (Shearing et Wood, 2003), sécurité nodale (Shearing, 2005) et réseaux de sécurité (Dupont, 2006). Ce programme théorique se fonde toutefois davantage sur des concepts que sur des faits solides. Mise à part la notable exception qu'est Dupont (2006), les données empiriques présentées à l'appui sont généralement sélectives, incomplètes ou anecdotiques. Dans la majorité des cas, l'hypothèse de départ concernant la "prolific expansion of private security in recent years ${ }^{16} \gg$ (O'Reilly et Ellison, 2006: 643) provient d'un rapport de De Waard (1999). Pourtant, ce dernier est moins catégorique qu'on le laisse paraitre: «the private security industry in Europe is the secondary source of protection, while it is the primary in North America and elsewhere [Australia and South Africa - the latter being the 'absolute champion'] ${ }^{17} \gg$ (De Waard, 1999: 169). En fait, pas un seul pays de l'Union européenne ne compte davantage d'agents de sécurité privée que d'agents de police publique. Le ratio sécurité privée/police publique (par 100000 habitants) favorise toujours cette dernière, particulièrement dans les pays méditerranéens: la Grèce $(0,05)$, l'Italie $(0,16)$, l'Espagne $(0,28)$, la France $(0,31)$, le Portugal $(0,35)$, de même que l'Autriche $(0,21)$, pays

15. «alors que la haute police publique protège l'État contre la subversion, la haute police privée protège contre la subversion du client» (traduction).

16. «l'expansion prolifique de la sécurité privée dans les dernières années» (traduction).

17. «l'industrie de la sécurité privée en Europe continue d'être une source secondaire de protection, alors qu'elle est la principale en Amérique du Nord et ailleurs [en Australie et en Afrique du Sud, cette dernière étant la championne toutes catégories]» (traduction). 
non méditerranéen (De Waard, 1999: 156, tableau VII). La forte expansion du nombre d'agents de sécurité privée se limite, pour l'instant, aux pays anglo-saxons et africains, particulièrement l'Afrique du Sud. Pour ce qui est du Royaume-Uni, le nombre d'agents de sécurité privée y dépassera bientôt celui des policiers professionnels (Crawford, 2003 : 149). Toutefois, toutes ces comparaisons statistiques sont trompeuses, car elles comparent deux catégories de personnel de sécurité aux fonctions et aux pouvoirs très différents (Nalla et Newman, 1991). De plus, les pays où la sécurité privée domine par le nombre ne sont pas ceux d'où provient le modèle de haute police.

Dans les pays anglo-saxons, la majorité des agents de sécurité privée sont impliqués dans des activités de basse police, comme l'illustrent bien les deux exemples suivants: (i) Pour une étude empirique sur l'enquête policière sur les homicides (Brodeur, 2005b), j'ai eu accès à tous les dossiers d'homicides de l'une des plus importantes forces policières urbaines du Canada et j'ai construit une banque de données constituée de 153 affaires classées entre 1990 et 2001. Dans toutes ces affaires, jamais un agent privé n'a joué de rôle, cette constatation étant par ailleurs validée par une étude exhaustive de la littérature sur les enquêtes d'homicide. (ii) Au Canada, le contrôle des voyageurs aériens et de leurs bagages a longtemps été assuré par des agences de sécurité privée. Or, à la suite des attaques du 11 septembre 2001, le gouvernement canadien a créé l'Administration canadienne de la sûreté du transport aérien (ACSTA), pour laquelle je suis consultant. Son mandat était de prendre en charge le travail des agences de sécurité privée, qui ne jouissaient pas de la confiance du public. À la lumière de ces études de cas, je voudrais proposer l'hypothèse suivante: plus les enjeux de sécurité seront élevés, plus le gouvernement (ré)affirmera ses responsabilités. Cette hypothèse repose sur deux considérations souvent négligées par les théoriciens de la sécurité privée: la légitimité, nécessaire à toute offre de services de sécurité, et l'autorité symbolique. Ces considérations sont généralement ignorées dans le paradigme de gouvernance nodale qui, à tort, met tous les fournisseurs de sécurité («nodes» ou «nœuds») sur un pied d'égalité.

B. Le continuum de la baute police. Tel qu'il a été mentionné précédemment, l'amalgamation des pouvoirs législatif, judiciaire et exécutif est une caractéristique de la haute police. Joseph Fouché, père de la haute police et ministre de la Police sous Napoléon, était aussi responsable 
du système pénitentiaire français. En 1808, il décrivait à l'Empereur les diverses catégories de personnes incarcérées en France suivant «une mesure de haute police». Parmi elles, se trouvaient des "prévenus n'ayant pas subi leur procès ni même ayant été traduits devant le tribunal par crainte de les voir acquittés par manque de preuve légale ${ }^{18}$ » (paraphrase). Récemment, le Parlement européen s'est prononcé dans un rapport sur «[1']utilisation alléguée de pays européens par la CIA pour le transport et la détention illégale de prisonniers» (Parlement européen, 2006). Le rapport affirme que le programme étatsunien de restitutions extraordinaires (extraordinary renditions):

$[\ldots]$ est une pratique extrajudiciaire en vertu de laquelle un individu soupçonné d'être impliqué dans des activités terroristes est illégalement enlevé, arrêté et/ou remis aux mains d'agents des États-Unis et/ou transporté vers un autre pays pour y subir un interrogatoire qui, dans la majorité des cas, implique une détention au secret et des actes de torture. (Parlement européen, 2006: paragraphe 27)

On y dit également qu'au moins 1245 vols opérés par la CIA ont survolé l'espace aérien européen ou fait escale dans des aéroports européens (Parlement européen, 2006: paragraphe 32). Autre exemple, Maher Arar, un citoyen canadien, a été arrêté à New York le 26 septembre 2002 par le FBI, alors qu'il revenait de vacances en Tunisie, sur la foi de renseignements erronés fournis par la GRC. Il a été transféré par les autorités étatsuniennes dans une prison syrienne, où il été questionné et torturé du 9 au 22 octobre 2002, et ce, sans droit de communication avec le monde extérieur. C'est grâce aux héroïques efforts de son épouse qu'il fut enfin libéré le 5 octobre 2003. Le gouvernement canadien a mis sur pied une commission d'enquête afin de faire la lumière sur cette affaire (Canada, 2006a et b). Il a été conclu que M. Arar était innocent de toutes les accusations portées contre lui et, en janvier 2007, il a reçu une somme compensatoire de l'ordre de 10 millions de dollars. Depuis, le gouvernement canadien a décidé d'enquêter sur la restitution à l'Égypte et à la Syrie de deux autres Canadiens, MM. El Maati et Almaki.

L'on peut tirer deux leçons de ces exemples. Premièrement, ils montrent que le fait d'assimiler les activités de haute police à ce qu'on pourrait appeler une activité clandestine de connaissance - c'est-à-dire

18. Cité dans Madelin (1930: 502; manuscrits originaux de Fouché, AFiv, 1314 et AFiv, 1320, n ${ }^{\circ} 20$ ). 
l'expertise-conseil et les enquêtes secrètes - est beaucoup trop réducteur. Aux États-Unis, en vertu d'un régime de guerre illusoire proclamé par l'administration Bush, la haute police jouit de tous les pouvoirs: elle donne des ordres exécutifs secrets parfaitement illégaux, garde des suspects en détention préventive illimitée, les fait juger par des commissions apparentées à la Chambre étoilée (Star Chamber) médiévale et applique les peines dans les conditions les plus punitives. Deuxièmement, il y a, dans cette idée que l'État est en perte de vitesse, une sorte de résurgence de l'utopie néo-marxiste, où la gouvernance nodale remplacerait la dictature du prolétariat. En fait, l'État n'a jamais été aussi arrogamment unilatéral que le sont aujourd'hui les États-Unis, le Royaume-Uni et le Canada.

C. Le partage d'information. Je mentionnerai brièvement que les réseaux de sécurité hybrides font face à davantage d'obstacles dans le partage de l'information que les réseaux publics. Selon Lippert et O'Connor (2006: 64), les firmes de sécurité privée partagent l'information de façon hautement sélective, entre elles, ainsi qu'avec la police publique: "They share intelligence with the public police when their clients are private citizens, but largely exclude the public police from the network when their clients are corporate ${ }^{19}$.» Ocqueteau (1997) a constaté le même phénomène en France, en soulignant que les agences de sécurité internes considéraient toute information sur leur clientèle comme étant confidentielle.

\section{Résonance symbolique}

Auparavant, l'agence de haute police du Canada était intégrée à une force de police - la GRC - tout comme celle des États-Unis fait maintenant partie d'une organisation policière - le FBI. Dans la seconde moitié du siècle dernier, le gouvernement canadien a créé plusieurs commissions pour déterminer si son service de sécurité devait demeurer dans le giron de la GRC ou s'il devait en faire une agence de haute police civile indépendante. Le gouvernement a finalement choisi cette dernière option en 1984, suivant un argument formulé par la commission Mackenzie: comme un service de sécurité doit inévitablement être impliqué dans des opérations clandestines contraires à la loi, voire aux

19. «Elles partagent des informations avec la police publique lorsque leurs clients sont des citoyens, mais excluent largement la police publique du réseau quand leurs clients sont des compagnies.» (Traduction) 
droits de la personne, sa mission est incompatible avec celle d'une organisation policière (Canada, 1969: 21).

La police est le symbole le plus manifeste de la loi (par exemple, les anglo-saxons se réfèrent aux policiers, dans le langage familier, par l'expression «the law»). Ceci est particulièrement vrai pour ce qui est des policiers affectés à des fonctions de basse police, tels les patrouilleurs en uniforme, qui utilisent leur visibilité pour produire des effets dissuasifs et rassurants. Pour leur part, les agences de haute police symbolisent le pouvoir de l'État, parfois dans ses aspects les plus arbitraires. Contrairement à la police, les services de renseignement basent leur pouvoir symbolique sur leur clandestinité, dans un monde de rumeurs, d'insinuations et de peur. Néanmoins, la littérature scientifique sur la police a montré à quel point la profession est «ternie» (Bittner, 1990 : $100)$ et que les policiers sont souvent eux aussi impliqués dans de «sales besognes» (Waddington, 2005: 376). Cette contradiction se dissout lorsque nous faisons la distinction entre la réalité et le symbole. Malgré le fait que la police, en réalité, enfreint souvent la loi, on ne peut accorder une légitimité symbolique à ces écarts, sous peine de briser le lien de confiance entre la police et les citoyens (Manning, 2003 : 3, 11-12). La résonance symbolique des agences de haute police est en fait tout le contraire: le non-respect des lois est le fondement même de leur mythologie. La culture de rebelle véhiculée par la fiction et les médias veut que l'efficacité d'une agence de renseignement soit proportionnelle à son non-respect des règles; on critique même les agents trop pointilleux du respect de la loi (United States, 2002: 53). Toutefois, cette culture d'illégalité institutionnalisée ne mine pas la légitimité de la haute police, et ce, pour deux raisons: premièrement, parce qu'elle est couverte, implicitement ou explicitement, par l'autorité de l'État (par exemple, l'assassinat d'opposants d'Israël, par ses services secrets); deuxièmement, parce qu'il est justifié, au champ de bataille, d'utiliser les mêmes tactiques que l'ennemi pour le vaincre ${ }^{20}$.

Où la haute police privée s'insère-t-elle dans cette structure symbolique? À mon avis, la police privée, qu'elle soit haute ou basse, est en

20. Par sa nature extrême, la torture illustre bien le gouffre infranchissable entre haute et basse police. Même pour les caïds du crime organisé, la torture n'a jamais été socialement acceptable ni acceptée. Il est donc impensable que des promoteurs de la torture, comme Dershowitz (2002) et Yoo (2006), qui ont toujours circonscrit leur position dans le contexte de la lutte anti-terroriste, auraient pu justifier leur position dans n'importe quel autre contexte sans se faire renier par leur université (Harvard et Berkeley, respectivement). 
déficit de pouvoir symbolique. De plus, si la haute police privée tentait un glissement vers l'extrémité coercitive du continuum, elle perdrait toute sa légitimité et ses agents s'exposeraient à des poursuites judiciaires. Mais ces hypothèses restent à confirmer. Ce qui est clair, cependant, c'est que la présence ou l'absence de ces dimensions symboliques de la haute police mériteraient de faire l'objet de plus amples recherches.

\section{Conclusion}

Ma discussion sur les caractéristiques de la haute police s'est concentrée sur ses aspects les moins étudiés jusqu'ici. La police d'absorption implique que l'usage du renseignement dans les causes criminelles est une entreprise épineuse qui se heurte souvent une forte résistance. L'amalgamation des pouvoirs, censés être séparés dans une démocratie, donne à la haute police un spectre de pouvoirs allant du travail du savoir jusqu'aux pratiques coercitives et aux opérations secrètes violentes. En faisant la distinction entre la protection du cadre institutionnel et constitutionnel d'un pays, d'une part, et la préservation d'un régime qui serait autrement rejeté, d'autre part, on peut considérer que la haute police n'est pas en contradiction avec la démocratie. Enfin, malgré l'utilisation croissante que fait la haute police des nouvelles technologies, l'utilisation d'infiltrateurs demeure le principal outil de surveillance de la haute police.

Loin de s'être estompée, la distinction entre haute et basse police a été accentuée de façon claire par toutes les commissions qui ont examiné le contexte policier dans lequel sont survenus les événements du 11 septembre 2001. Enfin, il est impératif, dans toute théorisation de la haute police, de tenir compte du développement de la haute police privée. Cependant, il est probable que la conceptualisation de la haute police ne sera que partiellement affectée par l'intégration de sa composante privée, puisque ses autres caractéristiques, comme son continuum renseignement-coercition, ne s'appliquent pas d'emblée à la haute police privée. De plus, il faudra aussi tenir compte de la structure symbolique de la haute police privée, qui est présentement lacunaire.

Finalement, il ne fait nul doute dans mon esprit que la distinction entre haute et basse police est instrumentale dans la compréhension de certaines pratiques élaborées dans le contexte de la soi-disant guerre au terrorisme. La restitution extraordinaire, l'interrogatoire extrême, la détention préventive indéfinie telle en vigueur à Guantanamo, ou encore 
cette ébauche de goulag transnational imposée par la CIA, sont autant de développements qui peuvent être examinés à travers la lorgnette de la théorie de la haute police. Dans un contexte plus large, le travail de Jonathan Simon (2007) sur la façon par laquelle les États-Unis se servent de la lutte contre le crime comme d'une stratégie de légitimation d'action et d'inaction gouvernementale et comme filtre des autres questions de gouvernance, trouve son application la plus littérale dans la guerre contre le terrorisme déclarée par l'administration Bush. Depuis le 11 septembre 2001, cette administration gouverne à travers le filtre $\mathrm{du}$ terrorisme, en utilisant des moyens de haute police.

\section{Références}

Anderson, M., den Boer, M., Cullen, P., Gilmore, W., Raab, C., \& Walker, N. (1995). Policing the European Union. Oxford: Clarendon Press.

Bayley, D. H., \& Shearing, C. (2001). The New Structure of Policing: Description, Conceptualization and Research Agenda. Washington, DC: US Department of Justice, National Institute of Justice.

Bittner, E. (1990). The functions of the Police in Modern Society: A Review of Background Factors, Current Practices, and Possible Role Models. In E. Bittner (Ed.), Aspects of Police Work (89-232). Boston: Northeastern University Press.

Breitel, C. D. (1980). Final Report of Special Master. New York: Political Rights Defence Fund.

Brodeur, J.-P. (1981). Legitimizing Police Deviance. In C. Shearing (Ed.), Organizational Police Deviance (127-160). Toronto: Butterworths.

Brodeur, J.-P. (1983/1996). High and Low Police: Remarks About the Policing of Political Activities. Social Problems, 30 (5), 507-521. Réédité dans R. Reiner (Ed.), (1996). Police Discretion and Accountability, Policing Vol. II (261-274). Aldershot (R.-U.): Dartmouth Pub.

Brodeur, J.-P. (2000/2005a). Cops and Spooks: the Uneasy Partnership. Police Practice and Research, 1 (3), 299-321. Réédité dans T. Newburn (Ed.), (2005). Policing: Key Readings (797-812). Cullompton, Devon: Willan Publishing.

Brodeur, J.-P. (2003). The Globalisation of Security and Intelligence Agencies: A Report on the Canadian Intelligence Community. In J.-P. Brodeur, P. Gill \& D. Töllborg (Eds.), Democracy, Law and Security. Internal Security Services in Contemporary Europe (210-261). Aldershot, R.-U.: Ashgate.

Brodeur, J.-P., \& Ouellet, G. (2005b). L'enquête criminelle. Criminologie, 38 (2), 39-64.

Brodeur, J.-P., \& Dupeyron, N. (2003). Democracy and Security: The French Intelligence Community. In J.-P. Brodeur, P. Gill \& D. Töllborg (Eds.), Democracy, Law and Security. Internal Security Services in Contemporary Europe (9-29). Aldershot, R.-U.: Ashgate. 
Brodeur, J.-P., \& Leman-Langlois, S. (2006). Surveillance Fiction or Higher Policing. In K. D. Happerty \& R. V. Ericson (Eds.), The New Politics of Surveillance and Visibility (171-198). Toronto: University of Toronto Press.

Canada (1969). Rapport de la Commision royale d'enquête sur la sécurité (Rapport Mackenzie). Ottawa: Imprimeur de la Reine.

Canada (2006a). Rapport sur les événements concernant Maber Arar: Les faits (vol. 1), Ottawa: Travaux publics et services gouvernementaux Canada, 3 volumes.

Canada (2006b). Un nouveau mécanisme d'examen des activités de la GRC en matière de sécurité nationale. Deuxième rapport. Ottawa: Travaux publics et services gouvernementaux Canada.

Comité de surveillance des activités de renseignement de sécurité (CSARS, 1994). L'Affaire du Heritage Front: rapport présenté au Solliciteur général du Canada. Ottawa: Comité de surveillance des activités de renseignement de sécurité.

Dershowitz, A. M. (2002). Why Terrorism Works. Understanding the Threat, Responding to the Challenge. Londres: Yale University Press.

De Waard, J. (1999). The Private Security Industry in International Perspective. European Journal on Criminal Policy and Research, 7, 143-174.

Dupont, B. (2006). Delivering Security through Networks: Surveying the Relational Landscape of Security Managers in an Urban Setting. Crime, Law and Social Change, 45, 165-184.

Funder, A. (2003). Stasiland. True Stories from Bebind the Berlin Wall. Londres: Granta Books.

Johnson, S. (1806). A Dictionary of the English Language. Londres, vol. II.

Johnston, L. (1992). The Rebirth of Private Policing. Londres: Routledge.

Lenoir, J. C. P. (1779). Mémoire sur la police en France, et particulièrement sur la police de Paris, présenté à la reine de Naples (seconde version) et présenté dans sa première version à l'impératrice d'Autriche. Document manuscrit, University Library of Cambridge, Rare Books Department, Classmark, Add. 4651.

L'Heuillet, H. (2001). Basse politique, haute police. Paris: Fayard.

Lippert, R., \& O'Connor, D. (2006). Security Intelligence Networks and the Transformation of Contract Private Security. Policing and Society, 16 (1), 50-66.

Madelin, L. (1930). Fouché, 1759-1820 (2 volumes). Paris: Librairie Plon.

Manning, P. K. (2003). Policing Contingencies. Chicago: The University of Chicago Press.

Manning, P. K. (2005). The Study of Policing. Police Quarterly, 8 (1), 23-43.

Marenin, O. (1982). Parking Tickets and Class Repression: the Concept of Policing in Critical Theories of Criminal Justice. Contemporary Crises, 6, 241-266.

Marenin, O. (1996). Policing Change, Changing Police: Some Thematic Questions. In O. Marenin (Ed.), Policing Change, Changing Police. International Perspectives (3-18). New York: Garland Publishing.

Marx, G. T. (1974). Thoughts on a Neglected Category of Social Movement Participants: Agents Provocateurs and Informants. American Journal of Sociology, 80 (2), 402-42. 
Marx, G. T. (1988). Undercover. Police Surveillance in America. Londres: University of California.

Nalla, M., \& Newman, G. (1991). Public versus Private Control: A Reassessment. Journal of Criminal Justice, 19, 537-547.

Naudé, G. (1639/1988). Considérations politiques sur les coups d'État. Paris: Éditions de Paris.

National Centre for Police Excellence (2005). Guidance on the National Intelligence Model 2005. Bedford, R.-U.: Centrex (produit pour l'Association des chefs de police).

Ocqueteau, F. (1997). Les défis de la sécurité privée. Paris: l'Harmattan.

O’Reilly, C., \& Ellison, G. (2006). Eye Spy Private High. Reconceptualizing High Police Theory. British Journal of Criminology, 46 (1), 641-660.

Parlement européen (2006). Projet de rapport interimaire sur l'utilisation alléguée de pays européens par la CIA pour le transport et la détention illégale de prisonniers (rapporteur G.C. Fava). Bruxelles: Commission temporaire sur l'utilisation alléguée de pays européens par la CIA pour le transport et la détention illégale de prisonniers.

Québec (1981a). Rapport de la Commission sur des opérations policières en territoire québécois (Rapport Keable). Québec: ministère des Communications.

Québec (1981b). Rapport sur les événements d'octobre 1970 (rapport de J.-F. Duchaîne). Québec: gouvernement du Québec, ministère de la Justice.

Reiner, R. (1994). Policing and the Police. In M. Maguire, R. Morgan \& R. Reiner (Eds.), The Oxford Handbook of Criminology (705-772). Oxford: Clarendon Press.

Shearing, C. (2005). Nodal Security. Police Quarterly, 8 (1), 57-63.

Shearing, C., \& Stenning, P. (1981). Modern Private Security: Its Growth and Implications. Crime and Justice, An Annual Review of Research, 3, 193-245.

Shearing, C., \& Wood, J. (2003). Nodal Governance, Democracy, and the New "Denizens". Journal of Law and Society, 30, 400-419.

Simon, J. (2007). Governing Through Crime. How the War on Crime Transformed American Democracy and Created a Culture of Fear. Oxford: Oxford University Press.

United States (2002). Congress, Senate, Select Committee on Intelligence. Additional Views of Senator Richard C. Shelby, Vice Chairman, Senate Select Committee on Intelligence: September 11 and the Imperative of Reform in the U.S. Intelligence Community. Washington, DC: Congress.

United States (2004). National Commission on Terrorist Attacks upon the United States. The 9/11 Commission Report: Final Report of the National Commission on Terrorist Attacks upon the United States. New York: Norton.

Waddington, P. A. J. (2005). Police (Canteen) Sub-Culture: an Appreciation. In T. Newburn (Ed.), Policing: Key Readings (364-385). Cullompton, Devon: Willan Publishing. (Paru pour la première fois en 1999).

Weiss, R. P. (1986). Private Detective Agencies and Labour Discipline in the United States. Historical Journal, 29 (4), 565-586. 
Yoo, J. (2006). War by Other Means: An Insider's Account of the War on Terror. New York: Atlantic Monthly Press.

Zaccardelli, G. (2005). Speaking Notes for a Presentation on Intelligence-Led Policing at the Canadian Association of Chiefs of Police Conference. Ottawa, 23 août 2005, GRC. Page disponible: www.rcmp-grc.gc.ca/speeches/sp_cacp_3_e.htm.

\begin{abstract}
The distinction between high and low policing is increasingly relevant in the wake of the terrorist attacks of 11 September 2001. This paper reviews the content of the high policing paradigm and addresses recent criticism. Its first part provides an update of the defining features of high policing: absorbent policing, power conflation favouring the executive, the primacy of protecting the state and massive use of infiltration through of covert informants. It is, thereafter, argued that the high and low police distinction is considered to run deeper than anticipated by the various bodies reporting on the policing and intelligence failure to prevent 9/11. In part three, the place of private security agencies in high policing is assessed. Private high policing must be taken into account, but it only shares in some of the defining features of high policing and is lacking in others. Finally, the contrast between high and low policing is examined in relation to symbolic significance.
\end{abstract}

KEYWORDS - High policing, low policing, political police, criminal police, inquiry commission.

RESUMEN - La distinción entre la alta policía (policía política) y la baja policía (la criminal, la ordinaria) ha adquirido mayor pertinencia luego de los atentados terroristas del 11 de septiembre de 2001 (9/11). El presente texto retoma el contenido del paradigma de la alta policía propuesto en 1983 y responde a algunas de las críticas que se le han hecho recientemente. Nos proponemos, de inicio, una actualización de las características de la alta policía. Este tipo de policía se considera como una policía de absorción, un amalgama de poderes al servicio del poder ejecutivo, de la protección del Estado y de infiltración de la sociedad por medio de informantes. Mostramos a continuación que las diferentes comisiones de investigación que han abordado las lagunas de acción policiaca de los servicios de información en la prevención de los atentados del 11 de septiembre han encontrado que la distinción entre la alta y la baja policía resultó mucho más profunda que lo que se había previsto. En la tercera parte del texto se aborda la cuestión de la existencia de una alta policía privada. Aunque la existencia de una alta policía privada no pueda ahora ponerse en duda, ésta no tiene más que una parte de las características que definieron el modelo inicial de la alta policía pública. En la última parte se analiza el contraste entre la alta y la baja policía, a la luz de su importancia simbólica.

Palabras Clave - Alta policía, baja policía, policía política, policía judicial, Comisiones de investigación. 